\title{
AVE isquêmico em paciente jovem sem fatores de risco: relato de caso
}

\author{
Ischemic stroke in young patient without risk factors: case report
}

Isadora Dias Lacerda ${ }^{1}$, Josué da Silva Brito ${ }^{1}$, Daniel Lima Souza ${ }^{1}$, Wesley Lobo Costa Júnior ${ }^{2}$, Talitha Araújo Faria ${ }^{3}$

Lacerda ID, Brito JS, Souza DL, Costa Júnior WL, Faria TA. AVE isquêmico em paciente jovem sem fatores de risco: relato de caso / Ischemic stroke in Young patient without risk factors: case report. Rev Med (São Paulo). 2018 maio-jun.;97(3):361-7.

RESUMO: Acidente vascular encefálico (AVE) caracterizase por um complexo de sintomas decorrentes de alterações no suporte sanguíneo cerebral, que duram pelo menos 24 horas causam lesões cerebrais. Pode ser classificado em isquêmico (AVEi) e hemorrágico (AVEh), sendo que o mais comum deles é o AVEi, caracterizado por interrupção do fluxo sanguíneo em uma determinada área do encéfalo devido a obstrução arterial ou venosa. A falta de suprimento sanguíneo causa um infarto na área vascularizada pelo vaso obstruído, causando morte neuronal. Existem fatores de risco modificáveis e não modificáveis para o AVE, sendo alguns deles: hipertensão, diabetes, tabagismo, etilismo, sedentarismo, dislipidemias, idade superior aos 55 anos, raça negra, história familiar e AVE prévio. O diagnóstico segue protocolos de acordo com o tempo de início dos sintomas, e basicamente, avalia-se quadro clínico, TC de crânio sem contraste ou ressonância magnética de crânio e exames laboratoriais. $\mathrm{O}$ tratamento varia de acordo com sua classificacão. No caso apresentado a paciente do sexo feminino, sem comorbidades prévias e fatores de risco, desenvolveu um AVEi mesencefálico com graves sequelas. Não foram investigados alterações em genes da coagulação que pudessem explicar a causa da patologia. $\mathrm{O}$ AVEi jovem consiste no acidente vascular encefálico presente em adultos com menos de 45 anos. A patologia necessita, além das investigações padrões, de uma averiguação quanto a etiologia genética. Várias patologias estão associadas ao quadro, como CADASIL, doença de Fraby, coagulopatias, anemia de células falciformes, síndrome de Sneddon, MELAS, síndrome de EhlersDanlos tipo IV, neurofibrimatose tipo 1 e doença de Moya-Moya. Diante disso evidencia-se a necessidade de se conhecer sobre novos estudos e atualizações que descrevam causas não tão conhecidas de AVE.

Descritores: Acidente vascular cerebral; Pacientes; Adulto jovem; Adulto; Fatores de risco.
ABSTRACT: Encephalic stroke (stroke) is characterized by a complex of symptoms resulting from changes in brain blood support, which last at least 24 hours cause brain damage. It can be classified as ischemic stroke and hemorrhagic stroke, the most common being ischemic stroke, characterized by interruption of blood flow in a certain area of the brain due to arterial or venous obstruction. The lack of blood supply causes an infarct in the area vascularized by the obstructed vessel, causing neuronal death. There are modifiable and non-modifiable risk factors for stroke, including hypertension, diabetes, smoking, alcoholism, sedentary lifestyle, dyslipidemias, age over 55, black race, family history and previous stroke. The diagnosis follows protocols according to the time of onset of symptoms, and basically, it is evaluated the clinical picture, skull CT without contrast or magnetic resonance of the skull and laboratory tests. The treatment varies according to its classification. In the case presented to the female patient, without previous comorbidities and risk factors, she developed a mesencephalic ischemic stroke with severe sequelae. No alterations in coagulation genes that could explain the cause of the disease were investigated. The young ischemic stroke consists of the cerebrovascular accident present in adults under 45 years. Pathology requires, in addition to the standard investigations, an inquiry into the genetic etiology. Several pathologies are associated with the disease, such as CADASIL, Fraby's disease, coagulopathies, sickle cell anemia, Sneddon syndrome, MELAS, Ehlers-Danlos syndrome type IV, neurofibrinatosis type 1 and Moya-Moya disease. Therefore, it is necessary to know about new studies and updates that describe not well-known causes of stroke.

Keywords: Stroke; Patient; Young adult; Adult; Risk factors.

1. Faculdade Atenas, Paracatu, MG, BR. Acadêmicos de Medicina. ORCID: Lacerda ID - https://orcid.org/0000-0003-1228-4621; Brito JS - https://orcid.org/0000-0003-2142-3689; Souza DL - 0000-0002-6783-4523. Email: isadoradias_lacerda@hotmail.com, josuedasilvabrito1998@gmail.com, daniel.limasouza@gmail.com.

2. Faculdade Atenas, Paracatu, MG, BR. Médico-preceptor. ORCID: Costa Júnior WL - https://orcid.org/0000-0001-9485-5824. Email: wesley.jr@hotmail.com.

3. Faculdade Atenas, Paracatu, MG, BR. Professora. ORCID: Faria TA - https://orcid.org/0000-0002-4252-6794. Email: talithabio@ yahoo.com.br.

Endereço para correspondência: Rua alumínio, 1326, Bairro Esplanada. Paracatu, MG, BR. Email: isadoradias_lacerda@hotmail.com. 


\section{INTRODUÇÃO}

A cidente vascular encefálico (AVE) é uma doença cerebrovascular ocasionada por deficiência na irrigação do tecido nervoso, decorrente de alterações vasculares, no qual há um complexo de sintomas, que duram pelo menos 24 horas, e que consistem no desenvolvimento rápido de distúrbios clínicos da função cerebral devido a lesões cerebral ${ }^{1,2,3}$. O tecido nervoso é completamente dependente da circulação sanguínea, pois é através da glicose e oxigênio que chegam por meio da circulação, que as células nervosas mantêm seu metabolismo ativo ${ }^{3}$.

O AVE pode ser classificado, dependendo da sua causa, em dois tipos: isquêmico (AVEi) e hemorrágico (AVEh), sendo que ambos resultam em comprometimento da função cerebral ${ }^{1,3}$. O mais comum deles é o AVEi, que se caracteriza por interrupção do fluxo sanguíneo em uma determinada área do encéfalo devido a obstrução arterial ou venosa decorrente de embolo, trombo, estenose, aterosclerose ou ainda por redução do débito cardíaco e hipotensão arterial grave ${ }^{1,4,5}$. Se a interrupção do fluxo sanguíneo durar menos que 24 horas as disfunções são consideradas reversíveis e caracteriza o chamado Acidente Isquêmico Transitório (AIT) ${ }^{6}$. Se persistir por mais que 24 horas, a isquemia pode levar a disfunções definitivas no tecido cerebral, devido à morte neuronal ${ }^{3}$.

O AVEh resulta do extravasamento de sangue para dentro ou para o entorno das estruturas do SNC decorrente da ruptura de um aneurisma, por exemplo. Quando ocorre extravasamento de sangue para o parênquima cerebral caracteriza uma hemorragia intracerebral e quando extravasa para o espaço entre parênquima cerebral e a membrana aracnoide caracteriza uma hemorragia subaracnóidea ${ }^{3,5}$. Em ambos os casos a falta de suprimento sanguíneo causa um infarto na área vascularizada pelo vaso rompido, o que também leva a morte neuronal ${ }^{5}$.

Segundo a Organização Mundial de Saúde (OMS), o AVE é a segunda causa de morte em todo o mundo, sendo superado apenas pelas doenças cardíacas ${ }^{7}$. O Ministério da Saúde classifica o AVE como primeira causa de morte e incapacidade no Brasil, o que causa grande impacto social e econômico ${ }^{8}$. No país estima-se 2.231 milhões de pessoas com AVE e 568.000 com incapacidade grave, sendo em homens a incapacidade severa de $29,5 \%$ e nas mulheres de $21,5 \%$.

Ao longo dos últimos anos têm sido identificados fatores de risco modificáveis e não modificáveis para o AVE, o que auxilia na prevenção de tal patologia ${ }^{4}$. Dos riscos modificáveis, encontra-se: hipertensão, diabetes, tabagismo, etilismo, sedentarismo, dislipidemias, doenças cardiovasculares como fibrilação atrial, doença coronariana e uso de prótese valvar sintética, além do uso de anticoncepcionais, entre outros ${ }^{3,5}$. Já na classe de riscos não modificáveis estão: idade superior aos 55 anos, raça negra, história familiar positiva e AVE prévio $^{5,8}$.

Além dos fatores de riscos, existem ainda causas não menos frequentes que levam ao AVE, como níveis elevados de homocisteína e deficiência em genes relacionados ao processo de coagulação, sendo: fator $\mathrm{V}$ de Leiden, antitrombina III, gene de protrombina, gene da proteína $\mathrm{C}$ e gene da proteína $\mathrm{S}^{10}$. Mutações nesses genes podem causar um tipo de AVE chamado criptogênico ${ }^{2}$.

Os riscos, excetuando hipertensão arterial e fumo, comportam-se de forma diferente de acordo com o sexo, sendo possível interpretar um maior risco para mulheres. A análise individual de fatores de risco demonstra essa propensão feminina ao se observar que para diabéticos o risco relativo de AVE é por volta de 4 para homens e de 6 para mulheres. Dois fatores de destaque para o sexo, que corroboram esse risco, é o uso de ACO, considerado fator de riscos nas mulheres que fumam, com mais de 35 anos ou com história de hipertensão, e a migrânea, um fator de risco para homens e mulheres, porém que se traduz em mulheres fumantes que utilizam ACO como uma razão de chance de 34,4 para $\mathrm{AVEi}^{3}$.

As manifestações clinicas nos pacientes acometidos pelo AVE variam bastante e relacionam-se a topografia da lesão. Além das áreas de acometimento relacionadas as disfunções clinicas causadas, existem alterações comuns provocadas pelo AVE, sendo elas: hipotonia seguida de hipertonia, espasticidade, movimentos estereotipados, apraxia, agnosia, afasia, perda dos mecanismos de controle postural, diminuição ou perda da sensibilidade tátil, térmica e dolorosa, além de alterações comportamentais ${ }^{3}$.

Os métodos diagnósticos do AVE seguem protocolos de acordo com o tempo de início dos sintomas. Basicamente, avalia-se quadro clinico, tomografia computadorizada (TC) de crânio sem contraste ou ressonância magnética de crânio (RNM), exames laboratoriais, exames cardíacos como eletrocardiograma (ECG) e duplex de carótidas ${ }^{2,3}$. A TC sem contraste é amplamente mais usada devido a maior disponibilidade, menos tempo para realização e menor custo. ${ }^{2}$

O tratamento no AVE varia de acordo com sua classificação em isquêmico e hemorrágico ${ }^{6}$. Independente, porém, dessa classificação, é recomendado que todo o tratamento se inicia dentro de 60 minutos desde a admissão no serviço hospitalar. O tratamento pressupõe uma interação multiprofissional, guiada por um neurologista. Se faz essencial o monitoramento cardiovascular, iniciando suplementação de oxigênio se necessária. $O$ paciente deve ser adequadamente hidrato com solução salina isotônica, administrado por via intravenosa. Recomenda-se avaliar a indicação do suporte ventilatório. Neste manejo inicial é preciso prevenir causas adicionais e prevenir a hiperglicemia, mantendo em níveis de 80 a $140 \mathrm{mg} / \mathrm{dL}$. Não se recomenda também a redução da pressão arterial em pacientes com AVEi, exceto os que apresentam níveis elevados, com pressão arterial sistólica maior que 220 
mmHG ou diastólica maior que $120 \mathrm{mmHg}$. Também se faz necessário se manter a temperatura axilar abaixo de 37, $5^{\circ} \mathrm{C}$, utilizando-se, se necessário, antipiréticos. Em casos de hipertemia se preconiza a investigação de causas infecciosas ${ }^{11}$.

Quando o AVE é classificado como isquêmico a indicação é a terapia trombolítica, que tem por objetivo recanalizar imediatamente o vaso sanguíneo obstruído e restaurar o fluxo sanguíneo cerebral. Essa terapia é feita com uso do rtPA(IV 0,9 mg/kg até dose máxima de $90 \mathrm{mg} ; 10 \%$ da dose em bolus e o restante infundido em 60 minutos) que possui curta janela terapêutica de três horas do início dos sintomas, mas é importante para reduzir as incapacidades geradas pelo $\mathrm{AVEi}^{6}$. O tratamento é contraindicado para pacientes com uso de heparina nas últimas 48 horas e TPPT prolongado, história de hemorragia intracraniana, traumatismo cranioencefálico grave nos últimos 3 meses, glicemia $<50 \mathrm{mg} / \mathrm{dL}$ com reversão sintomática após correção, história de sangramentos gastrointestinal ou geniturinário e evidências de endocardite, gravidez e embolo séptico ${ }^{12}$. Nas primeiras 24 horas, o paciente deve ser mantido em jejum e não deve receber sonda nasoentérica. Outros procedimentos que devem ser preteridos para o paciente nas primeiras 24 horas são a punção arterial e a cateterização venosa central. Após 24 horas pós-trombólise é indicado se iniciar profilaxia contra trombose venosa profunda (TVP), com heparina de baixo peso ou enoxaparina, assim como se inicia o tratamento com antiagregante plaquetário e anticoagulantes ${ }^{10}$.

No AVEh não há terapia específica, o que o torna mais incapacitante e com maior taxa de mortalidade. Portanto, nesse caso, trata-se somente a pressão arterial (PA) se PAS (pressão arterial sistólica) $>180 \mathrm{mmHg}$ ou PAM (pressão arterial média) $>110 \mathrm{mmHg}$. Em casos de hipertensão craniana (HIC), monitoriza-se a pressão intracraniana (PIC) para manter PPC (pressão de perfusão cerebral) $\geq$ $60 \mathrm{mmHg}$. Em casos de hemorragia intraventricular que possa evoluir com hidrocefalia, recomenda-se a colocação de uma DVE (derivação ventricular externa) ${ }^{6}$.

O objetivo deste trabalho é descrever um caso em que uma paciente de 29 anos, sem nenhum fator de risco modificável ou não modificável, que foi diagnosticada com AVEi mesencefálico e prosseguiu com intensas sequelas. Estudos sobre AVE observam grandes sequelas em pacientes jovens com ausência de fatores de risco, visto que pouco se sabe sobre as condutas nesses casos. Diante disso, esse relato tem o intuito de elucidar problemáticas a respeito do assunto.

\section{RELATO DE CASO}

Paciente, 29 anos, sexo feminino, deu entrada no Pronto Socorro de atenção primária, da cidade de Paracatu-MG, apresentando quadro de rebaixamento do nível de consciência associado à hiperreflexia de membro inferior direito (MID) após cefaleia intensa há 01 dia. Paciente negava tabagismo, etilismo e uso de drogas ilícitas. Não fazia uso de anticoncepcional. Sem casos familiares de hipertensão arterial, dislipidemia e doenças cerebrovasculares. Ao exame físico apresentava pupilas isocóricas e fotorreagentes, com sinais negativos de meningite e Babinski bilateralmente. Pressão Arterial: 120x70mmHg, frequência cardíaca: 78 bpm. Foi solicitada avaliação neurológica na qual se questionou a hipótese diagnóstica de trombose do seio venoso. Foi realizada TC de crânio que se mostrou normal e punção liquórica cristalina e sem alterações. Com suspeita diagnóstica mantida, realizou-se RNM de crânio, porém o laudo da mesma mostrou área de restrição à difusão no mesencéfalo, sem realce ao meio de contraste e sem efeito expansivo significativo, além de ausência de fluxo no segmento distal da artéria basilar. Esse conjunto de achados foi compatível com evento vascular isquêmico agudo mesencefálico (AVEi mesencefálico), sendo optado por intubação orotraqueal e encaminhamento da paciente para suporte neurointensivo.

Paciente foi admitida no CTI intubada, sedada (RASS -5) em uso de midazolan $10 \mathrm{ml} /$ hora e fentanil $10 \mathrm{ml} /$ hora, além de clexane $60 \mathrm{mg}$ SC $12 / 12$ horas para profilaxia de TVP e omeprazol 40mg EV 24/24 horas para profilaxia de hemorragia digestiva alta (HDA), sob ventilação manual, corada, hidratada, afebril, acianótica, anictérica e hemodinamicamente estável. Apresentava pupilas isocóricas, fotorreativas e mióticas. Ao exame físico cardiovascular: Ritmo cardíaco regular em 2 tempos, bulhas normofonéticas sem sopros; respiratório: murmúrio vesicular fisiológico sem ruídos adventícios, sat. O2 100\% com uso de ventilação mecânica; neurológico: pupilas isocóricas e fotorreativas, mantendo hiperreflexia de MID. Foi solicitado revisão laboratorial e iniciado clexane 60 mg. Manteve-se sem intercorrências e dieta zero até o primeiro dia de internação, quando foi realizada passagem de sonda nasoentérica. Os resultados laboratoriais em 14/10/2016 foram: Hemoglobina (Hb): 13,5; Hematócrito

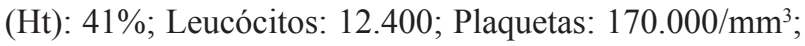
Tempo de ação da protrombina (TAP): $16,3 \mathrm{seg}$; Tempo de tromboplastina parcial ativada (TTPA): $32,7 \mathrm{seg}$; RNI: 1,34; Colesterol total: 140mg/dL; HDL: $66 \mathrm{mg} / \mathrm{dL}$; LDL: $74 \mathrm{mg} / \mathrm{dL}$.

No primeiro dia de internação, a paciente permaneceu sedada RASS -5, adaptada ao uso de ventilação mecânica e sem uso de drogas vasoativas. Encontrava-se hemodinamicamente estável, afebril, com bom padrão respiratório e sem sinais de infecção. Ao exame físico: regular estado geral, corada, hidratada, pupilas isocóricas e fotorreagentes com mantimento de hiperreflexia e tendência extensora dos membros. Exame físico cardiovascular e respiratório sem alterações. Mantido suporte intensivo com acompanhamento neurológico e início do desmame da sedação e ventilação mecânica. No mesmo dia foi reduzido a dose de midazolan para $7 \mathrm{ml} /$ hora e fentanil para 
$7 \mathrm{ml} /$ hora, corroborando com a conduta de desmame. No segundo dia, a paciente, ainda sob ventilação mecânica, se manteve sedada na CTI. Foi avaliada pela neurologia que detectou pupilas isocóricas, fotorreagentes, reagindo a dor com abertura ocular, movimentos ineficazes com tendência extensora dos quatro membros caracterizando uma tetra espasticidade. Estável ao ponto de vista da neurologia.

Em avaliação neurológica no terceiro dia da internação a paciente encontrava-se tetraparética com predomínio em grupo flexor nos membros à esquerda, com importante déficit motor bilateral (força $+/+++++$ ) e sedação mantida. Já no quarto dia de internação paciente apresentava-se hemodinamicamente estável, apresentando 3 picos febris: $38^{\circ} \mathrm{C}$ ás $19: 00,37,9^{\circ} \mathrm{C}$ às $01: 00$ horas e $37,8^{\circ} \mathrm{C}$ ás 03:00 horas. Permaneceu sedada com uso de fentanil e midazolan, e intubada sob ventilação mecânica com sat. 99\%. À gasometria: pH sanguíneo: 7,44; PCO2: 26; HCO3:17,7; $\mathrm{PaO} 2 / \mathrm{F} 1 \mathrm{O} 2:$ 413. Foi mantida sedação e feito reposição de $\mathrm{K}^{+}$. Um dia depois, foi solicitado hemograma que se mostrou alterado com leucocitose significante. Diante da clínica febril e dos resultados laboratoriais foi solicitado cultura. A paciente evoluiu até o nono dia de internação na CTI com picos febris, Glasgow estimado em 13, reagindo a dor com movimento de abertura ocular mecânica. Continuava a apresentar tetraparesia flácida acentuada à esquerda com predomínio nos flexores de braço e perna, simulava decerebração. Por isso foi iniciado ceftriaxone $2 \mathrm{~g} \mathrm{EV} \mathrm{24/24horas,} \mathrm{e} \mathrm{indicado}$ pela neurologia a suspensão da sedação e repetição da TC de crânio.

No dia seguinte apresentou 2 picos febris e a tentativa de desmame da sedação foi malsucedida, devido à dessincronização ventilatória importante. A paciente teve que ser traqueostomizada no dia $12^{\circ}$ dia, sem intercorrências. No quinto dia de antibioticoterapia, e $11^{\circ}$ dia de internação na CTI, foi substituído o ceftriaxone pelo meropenen guiado por cultura. Ainda sedada sob ação de fentanil e midazolan $10 \mathrm{ml} /$ hora, apresentou abalos musculares inespecíficos e 9 picos febris. Ao exame físico estava descorada. No dia seguinte foi acrescido vancomicina ao esquema de antibioticoterapia, solicitado urocultura e indicado desmame da sedação.

No dia 15 de internação a paciente evoluiu com Glasgow 14 respondendo ao chamado com abertura ocular parcial e apresentava comunicação e entendimento visual. No dia seguinte foi solicitado Doppler transcraniano pela neurologia e a paciente saiu da sedação, apresentando abertura ocular espontânea. O resultado do Doppler foi: fluxo normal em topografia de artérias cerebrais médias bilaterais com índices de resistência nos limites superiores da normalidade. O fluxo normal em topografia de artérias vertebrais com índices nos limites superiores da normalidade. Na profundidade de 90 a $94 \mathrm{~mm}$ em topografia de artéria basilar apresenta aumento da velocidade de fluxo com turbulência nessa região. A conclusão clínica foi um possível aumento da resistência vascular intracraniana, com sinais sugestivos de estenose segmentar moderada a grave em topografia de artéria basilar na profundidade de 90 a $95 \mathrm{~mm}$. No dia 20 de internação na CTI foram solicitados exames laboratoriais para pesquisar trombofilia, e no dia seguinte por continuar a apresentar clonus nos quatro membros decorrente da tetra espasticidade, foi iniciado Baclofeno 5mg 12/12 horas. Com 11 dias de internação, o esquema de meropenen foi suspenso e indicou-se desmame da ventilação mecânica.

No dia 24 de internação na CTI foi realizada prescrição pela neurologia a fim de cessar as contraturas musculares involuntárias: Hidantal 12/12 horas, Fenobarbital 12/12 horas e Baclofeno 10mg 12/12 horas. Com 32 dias de internação de CTI a paciente recebera alta para a clínica médica, onde permaneceu alguns dias para estabilização dos movimentos clônicos decorrentes da tetra espasticidade e avaliação do quadro neurológico geral.

$\mathrm{Na}$ enfermaria de clínica médica evoluiu sem intercorrência, com desmame da oxigenioterapia e decanulação, realização de fisioterapia motora e psicomotora. No $4^{\circ}$ dia de internação na clínica medica apresentou tumefação em terço distal da coxa bilateralmente sem sinais flogísticos, sendo solicitado USG de partes moles que não foi realizado por falha nos níveis de atenção regional. Com 14 dias de internação na clínica médica paciente evoluiu com melhora da espasticidade dos membros superiores e dos espasmos musculares após aumento da dose de Baclofeno 6/6 horas, respiração em ar ambiente sem desconforto respiratório, saturação de $97 \%$, e resposta a comandos com expressão facial de sorrisos.

No $15^{\circ}$ dia, paciente sequelada de AVE vertebrobasilar com lesão de tronco encefálico, apresentava pouca interação com o examinador, choro motivado, resposta verbal prejudicada, abertura ocular espontânea, pupilas isocóricas e fotorreagentes, nuca livre, oftalmoplegia do terceiro nervo à direita, tetraparesia espástica predominante à esquerda com contratura importante em região proximal de coxa.

A paciente teve alta da neurologia com indicação de fisioterapia e fonoterapia, prescrição de Baclofeno e Fenobarbital, orientação familiar quanto ao acompanhamento ambulatorial neurológico.

\section{DISCUSSÃO}

AVEi em adultos jovens, isto é, o acidente vascular cerebral que se dá em pacientes de 18 a 45 anos (estendível até 50 anos), é um evento dotado de excepcionalidade, o qual apresenta melhor prognóstico de sobrevida, não obstante, represente maior impacto socioeconômico, devido a maior taxa de morbimortalidade e sequelas físicas e emocionais que podem levar a inatividade de pessoa economicamente ativa ${ }^{13-15}$. 
Em pacientes menores de 45 anos, a incidência de AVE varia de $3,9 \%$ a $13,5 \%$ dos casos. Caracteristicamente, a incidência prepondera entre a sétima e oitava décadas de vida, quando já se apresentam alterações metabólicas e cardiovasculares relativas à idade $\mathrm{e}^{13,16}$.

O AVE em jovens requer, além das investigações etiológicas padrões - que incluem lipidograma, triglicerídeos séricos, ácido úrico sérico, glicemia em jejum, hemograma completo, urianálise, proteína $\mathrm{C}$ reativa, coagulograma, eletrocardiograma e exames de neuroimagem -, o levantamento de fatores genéticos e familiares, em caso de etiologia indeterminada, como o fator $\mathrm{V}$ de Leyden, antitrombina III, mutação da protrombina, proteínas S e C da coagulação ${ }^{10,13-15}$.

Um importante revés do AVE em jovens consiste na dificuldade de se determinar a etiologia. Essa permanece indeterminada em até $27 \%$ dos casos. Sendo, juntamente a alta taxa de incapacidade, a indeterminação um fortificante para a necessidade de se reforçar a prevenção primária ${ }^{16}$.

Já foram descobertas várias mutações e genes relacionados ao AVE jovem. Entre as principais patologias genéticas associadas ao acidente vascular estão: CADASIL, doença de Fraby, coagulopatias, anemia de células falciformes, síndrome de Sneddon, MELAS, síndrome de Ehlers-Danlos tipo IV, neurofibrimatose tipo 1 e doença de Moya-Moya ${ }^{17,18}$.

A arteriopatia cerebral autossômica dominante com infantes subcorticais e leucoencefalopatia (CADASIL) é a angiopatia cerebral hereditária mais comum, embora seja uma doença rara. Essa patologia consiste em uma mutação dominante no gene NOTCH3, presente no cromossomo $19 q 12$, que codifica um receptor transmembrana de células musculares lisas vasculares. O sinal por ele traduzido atua na manutenção da estabilidade estrutural e funcional da célula lisa vascular. As mutações resultam em anomalias vasculares, como alteração de tônus miogênico e de autorregulação do fluxo cerebral, que se apresenta diminuído. Existe ainda isquemia subcortical crônica, que pode causar infartos lacunares recorrentes e deformação estrutural ${ }^{17-21}$.

As manifestações da CADASIL se apresentam em $85 \%$ dos casos como AVE/AIT, que se dá por volta dos 45 anos, em pacientes fatores de risco para doenças cerebrovasculares. Em considerável parte dos casos, há manifestação de enxaqueca com áurea por volta dos 30 anos de idade ${ }^{17,20,22}$.

A doença de Fabry está associada ao gene GALA presente na região Xq22. Na patologia há um déficit parcial ou total da enzima alfagalactosidase A, resultando em acúmulo de glicotriaosilceramida no endotélio vascular, pericitos, células musculares lisas, células epiteliais, histiócitos e células do tecido conjuntivo. Esse acúmulo leva a protuberâncias ao lúmen que ocasionam obstrução que progride para isquemia ${ }^{17,23}$.

A doença de Fabry não apresenta o AVE como sua única manifestação clínica. A primeira variação se dá entre homozigotos e heterozigotas. Em homozigotos as manifestações vão de parestesias crônicas e episódios de dor abdominal, diminuição da sudorese, intolerância a calor, presença de angioqueratomas na pele e mucosas e comprometimentos cardíacos, renais e cerebrais. Em heterozigotas, há a variação de quadros assintomáticos aos tão severos quanto os homozigotos ${ }^{23}$.

As coagulopatias hereditárias são doenças hemorrágicas decorrentes do déficit funcional ou quantitativo de um ou mais fatores de coagulação. Uma das principais alterações em prevalência populacional consiste na presença do fator $\mathrm{V}$ de Leiden (presente em até $20 \%$ da população $)^{24,25}$. O fator V é uma glicoproteína prócoagulante. Quando na cascata de coagulação a trombina se liga ao trombomodulina há ativação da proteína $\mathrm{C}$. A proteína $\mathrm{C}$ cliva o fator $\mathrm{V}$, tornando-o inativo, permitindo-se assim a progressão normal para a fibrinólise. Na alteração do fator $\mathrm{V}$ de Leiden há resistência à clivagem pela proteína $C$ ativada, levando a exacerbação na formação da trombina e, consequentemente, da coagulação ${ }^{17,24,25}$.

Outras coagulopatias bastante abordadas pela a literatura associada ao AVE consistem no déficit de antitrombina III e mutação do gene da protrombina ${ }^{17,25}$.

A anemia falciforme, por sua vez, se associa a AVE jovens, porém, é mais conhecida para AVE em crianças da raça negra, fazendo-se presente em $75 \%$ dos $\operatorname{casos}^{26}$. A patogênese envolve a substituição de valina por ácido glutâmico da cadeia $\beta$ da hemoglobina. A hemoglobina $S$, menos solúvel, polimeriza-se resultando em deformação do eritrócito, que oclui vasa-vasorum. A oclusão resulta em proliferação da íntima e isquemia de grandes vasos, resultando na oclusão destes e no $\operatorname{AVE}^{17,25,27}$.

A síndrome de Snedonn apresenta sugestiva herança autossômica dominante, estando associada à livedo reticular e doenças cerebrovasculares. Sua etiologia genética é desconhecida, existindo história familiar em uma minoria de casos. Ela se associa ao anticorpo antifosfolipídio. A fisiopatologia da síndrome ainda não foi esclarecida ${ }^{17,28}$.

A miopatia mitocondrial, encefalopatia, acidose láctica e episódios de AVE (MELAS) é uma síndrome oriunda de alterações no DNA mitocondrial que se apresenta por enxaqueca com áurea recorrente, demência, crises epiléticas ${ }^{15}$. A síndrome apresenta variabilidade fenotípica. Os acidentes vasculares cerebrais associados a síndrome acontecem antes dos $40 \operatorname{anos}^{29,30}$.

A síndrome de Ehlers-Danlos tipo IV, também chamada de síndrome de Ehlens Danlos Vascular ou síndrome de Sack-Barabas, consiste em uma doença autossômica dominante, na qual há uma mutação no cromossomo 2, o que resulta em alterações estruturais do colágeno tipo III, levando a fragilidade vascular. Nessa síndrome há a possibilidade rotura arterial espontânea; principal responsável da mortalidade dos acometidos pela síndrome. Além dos sintomas vasculares, há alterações 
na pele, que se apresenta pálida e frágil, com hematomas espontâneos. A cicatrização de feridas é deficiente, insensibilidade ao frio e calor, ruptura de órgãos, como o cólon, aneurismas, rupturas de tendões, entre outros ${ }^{31,32}$.

A doença de von Recklinghausen ou neurofibromatose do tipo 1 consiste em uma facomatose autossômica dominante, na qual há mutação no gene NF1 presente no cromossomo 17. Essa patologia se manifesta por hemorragias intracerebrais espontâneas e por AVEi, mancha café-com-leite, neurofibromas dérmicos e plexiformes ${ }^{33-37}$. O portador da síndrome, além das complicações cerebrais desenvolve tumores benignos em taxas maiores do que a população normal ${ }^{33}$.

No Brasil, dois estudos contribuem para a compreensão do AVE em jovens. O estudo realizado em 2001 por Zétola acompanhou 164 pacientes de 15 a 49 anos, sendo observada a maior incidência aterotrombótica, sendo presentes os fatores de risco HAS, tabagismo, dislipidemia, DM e álcool ${ }^{13}$. Já o relato de Sousa-Pereira em 2010, encontrou 44 pacientes com menos de 50 anos, sendo que em pelo menos $84 \%$ tinha sido identificado um fator de risco cardiovascular, sendo considerado frequente o achado de dislipidemia no AVEi. A análise ainda observou como a terapêutica mais utilizada o antiagregante plaquetário AAS e com frequência o uso de varfarina ${ }^{15}$.

No presente caso, houve uma impossibilidade de se realizar uma investigação biomolecular e genética da paciente - metodologia necessária, visto que se ausentam fatores de risco clássicos e a idade inferior a 45 anos. Seria necessária também uma investigação aprofundada em relação as coagulopatias hereditárias, provável etiologia do caso relatado, visto a alteração em TAP, RNI e a destoante contagem normal de plaquetas. Salienta-se a necessidade de se realizar uma história genética em quadros de AVE jovem, a fim de se determinar uma terapêutica mais individualizada e se realizar ações preventivas, como a terapia de reposição através do uso, quase sempre, de plasma, e acompanhamento da evolução das manifestações gênicas ${ }^{10,17,25}$.

\section{CONCLUSÃO}

Diante dos estudos atualizados das prováveis causas de AVE, percebe-se que manifestações clínicas que levam a suspeita de AVE em pacientes jovens devem ser investigadas além dos exames de imagem, com investigação individualizada sobre alterações nos genes da coagulação.

Mesmo com graves sequelas e intuito de tratamento das mesmas, deve-se buscar a causa do AVE de forma primária, principalmente nos casos onde não se evidenciam fatores de risco.

Contribuição dos autores: Lacerda ID, Brito JS, Souza DL Planejamento, análise do caso e redação do artigo; Costa Júnior WL Orientador; Faria TA - Co-orientadora e revisora do artigo.

\section{REFERÊNCIAS}

1 Sacco RL, Kasner SE, Broderick JP. Caplan LR, Connors JJB, Culebras A. An updated definition of stroke of the 21s century. Stroke. 2013;44(7):2064-89. doi: 10.1161/ STR.0b013e318296aeca.

2 Rolim CLRC, Martins M. Qualidade do cuidado ao acidente vascular cerebral isquêmico no SUS. Cad Saúde Pública. 2011;27(11):2106-16. doi: 10.1590/S0102$311 X 2011001100004$.

3 Cancela DMG. O acidente vascular cerebral - classificação, principais consequências e reabilitação. Psicologia. 2008:118. Disponível em: http://www.psicologia.pt/artigos/textos/ TL0095.pdf.

4 Chaves MLF. Acidente vascular encefálico: conceituação e fatores de risco. Rev Bras Hipertens. 2000;7(4):37282. Disponível em: http://departamentos.cardiol.br/dha/ revista/7-4/012.pdf.

5 Almeida EO. Frequência e fatores relacionados à disfagia orofaríngea após acidente vascular encefálico [Dissertação]. Belo Horizonte: Instituto de Ciências Biológicas da Universidade Federal de Minas Gerais; 2009.

5 Oliveira RMC, Andrade LAF. Acidente Vascular Cerebral. Rev Bras Hipertens. 2001;8(3):280-90. Disponível em: http:// departamentos.cardiol.br/dha/revista/8-3/acidente.pdf.

6 Figueiredo MM, Bichuetti DD, Gois AFT. Evidências sobre diagnóstico e tratamento do acidente vascular encefálico no serviço de urgência. Diagn Tratamento. 2012;17(4):167-72. Disponível em: http://files.bvs.br/upload/S/1413-9979/2012/ v17n4/a3328.pdf.

7 Organização Mundial da Saúde. Manual STEPS de acidentes vascular cerebrais da OMS: enfoque passo a passo para a vigilância de acidentes vascular cerebrais. Genebra: WHO Press; 2006. Disponível em: http://www1.paho.org/hq/ dmdocuments/2009/manualpo.pdf.

8 Brasil. Acidente vascular cerebral (AVC) [citado 10 nov. 2017]. Disponível em: http://www.brasil.gov.br/ saude/2012/04/acidente-vascular-cerebral-avc.

9 Bensenor IM, Goulart AC, Szwarcwald CL, Vieira MLFP, Malta DC, Lotufo PA. Prevalence of stroke and associated disability in Brazil: National Health Survey - 2013. Arq Neuro-Psiquiatr. 2015;73(9):746-50. doi: 10.1590/0004$282 X 20150115$.

10 Brasil. Ministério da Saúde. Secretaria de Atenção à Saúde. Departamento de Atenção Especializada. Manual de rotinas para atenção ao AVC. Brasília: Editora do Ministério da Saúde; 2013. Disponível em: http://bvsms.saude.gov.br/bvs/ publicacoes/manual_rotinas_para_atencao_avc.pdf.

11 Martins SCO, Freitas GR, Pontes-Neto OM, Pieri A, Moro $\mathrm{CHC}$, Jesus PAP, et al. Guidelines for acute ischemic stroke treatment: part II: stroke treatment. Arq Neuro-Psiquiatr. 2012;70(11):885-93. doi: 10.1590/S0004-282X20120 01100012 .

12 Oliveira-Filho J, Martins SCO, Pontes-Neto OM, Longo A, 
Lacerda ID, et al. AVE isquêmico em paciente jovem sem fatores de risco.

Evaristo EF, Carvalho JJF, et al. Guidelines for acute ischemic stroke treatment: part I. Arq Neuro-Psiquiatr. 2012;70(8):6219. doi: 10.1590/S0004-282X2012000800012.

13 Zétola VHF, Nóvak EM, Camargo CHF, Carraro Júnior H, Coral P, Muzzio JA, et al. Acidente vascular cerebral em pacientes jovens: análise de 164 casos. Arq NeuroPsiquiatr. 2001;59(3B):740-5. doi: 10.1590/S0004282X2001000500017.

14 Tavares TM. Acidente vascular encefálico em adultos jovens: revisão da literatura [TCC]. Goiânia: PUC; 2011. Disponível em: https://repositorio.ufsc.br/xmlui/ handle/123456789/170574.

15 Sousa-Pereira SR, Braga CA, Garcia EC, Teixeira, AL. Acidente vascular encefálico em adultos jovens: análise de 44 casos. Rev Med Minas Gerais. 2010;20(4):514-8. Disponível em: http://rmmg.org/artigo/detalhes/332.

16 Cardoso T, Fonseca T, Costa M. Acidente vascular cerebral no adulto jovem. Acta Med (Port). 2003;16:239-44. Disponível em: https://www.actamedicaportuguesa.com/revista/index. php/amp/article/viewFile/1189/841.

17 Guimarães J, Azevedo E. Causas genéticas de acidente vascular cerebral isquémico. Arq Med (Porto). 2010;24(1):23-8. Disponível em: http://www.scielo. mec.pt/scielo.php?script $=$ sci_arttext\&pid $=\mathrm{S} 0871$ $34132010000100004 \& \operatorname{lng}=$ pt.

18 Lindgren A. Stroke genetics: a review and update. J Stroke. 2014;16(3):114-23. doi:10.5853/jos.2014.16.3.114.

19 Hawkes MA, Wilken M, Bruno V, Pujol-Lereis V, Povedano G, Saccoliti M, Taratuto A, Ameriso SF. Cerebral autosomal dominant arteriopathy with subcortical infarcts and leukoencephalopathy (CADASIL) in Argentina. Arq Neuropsiquiatr. 2015;73(9):751-4. doi: 10.1590/0004$282 X 20150113$.

20 Opherk C, Gonik M, Duering M, Malik R, Jouvent E, Hervé $\mathrm{D}$, et al. Genome-wide genotyping demonstrates a polygenic risk score associated with white matter hyperintensity volume in CADASIL. Stroke. 2014;45(4):968-72 . doi: 10.1161/ STROKEAHA.113.004461.

21 Correia NMF. Síndrome CADASIL: epidemiologia, clínica, genética [Dissertação]. Porto: Instituto de Ciências Biomédicas Abel Salazar; 2011.

22 Hawkes MA, Wilken M, Bruno V, Pujol-Lereis V, Povedano G, Saccoliti M et al . Cerebral autosomal dominant arteriopathy with subcortical infarcts and leukoencephalopathy (CADASIL) in Argentina. Arq Neuro-Psiquiatr. 2015;73(9):751-4. Doi: 10.1590/0004$282 X 20150113$.

23 Boggio P, Luna PC, Abad ME, Larralde M. Doença de Fabry. An Bras Dermatol. 2009;84(4):367-76. http://dx.doi. org/10.1590/S0365-05962009000400008.

24 Correia ALF. Factores genéticos de risco para acidente vascular jovem [Dissertação]. Aveiro: Universidade de Aveiro; 2011. Disponível em: https://ria.ua.pt/bitstream/10773/7925/1/ FACTORES\%20GEN\%C3\%89TICOS\%20DE\%20 RISCO\%20PARA\%20AVC\%20JOVEM.pdf.
25 Brasil. Ministério da Saúde. Secretaria de Atenção à Saúde. Departamento de Atenção Especializada. Manual de tratamento das coagulopatias hereditárias. Brasília: Editora do Ministério da Saúde; 2006. Disponível em: http://bvsms. saude.gov.br/bvs/publicacoes/06_1132_M.pdf.

26 Caridade S, Machado A, Ferreira C. Acidente vascular cerebral em doente com anemia de células falciformes. Arq Med. 2007;21(5-6):155-7. Disponível em: ww.scielo.mec.pt/ pdf/am/v21n5-6/v21n5-6a05.pdf.

27 Simões AF, Garcia P, Fernandes I, Ventura L, Silva R, Barata D. Anemia de células falciformes e acidente vascular cerebral: uma história evitável. Acta Med Port. 2011;24(4):637-40.

28 Dutra LA, Braga-Neto P, Pedroso JL, Barsottini OGP. Síndrome de Sneddon: relato de caso e revisão sobre a relação com a síndrome do anticorpo antifosfolipídio. Einstein (São Paulo). 2012;10(2):230-2. Disponível em: http://www.scielo. br/pdf/eins/v10n2/pt_a18v10n2.pdf.

29 Lorenzoni PJ, Werneck LC, Kay CSK, Silvado CES, Scola R. When should MELAS (Mitochondrial myopathy, Encephalopathy, Lactic Acidosis, and Stroke-like episodes) be the diagnosis? Arq Neuro-Psiquiatr. 2015;73(11):959-67. doi: 10.1590/0004-282X20150154.

30 Scaglia F, Northrop JL. The mitochondrial myopathy encephalopathy, lactic acidosis with stroke-like episodes (MELAS) syndrome: a review of treatment options. CNS Drugs. 2006;20(6):443-64. Erratum in: CNS Drugs. 2008;22(1):81.

31 Silva JFB. Síndrome de Ehlers-Danlos Vascular (SEDV), antes llamado SED tipo IV. Rev Chil Reumatol. 2008;24(1):6-11. Disponivel em: http://www.sochire.cl/ bases/r-341-1-1343705929.pdf.

32 Belczak SQ, Klajner RK, Ogawa LC, Lucato LL, Zeque BS, Macedo FB et al. Tratamento endovascular de aneurisma de tronco braquiocefálico em paciente com síndrome de Ehlers-Danlos tipo IV. J Vasc Bras. 2016;15(3):239-44. Doi: 10.1590/1677-5449.001016.

33 Souza JF, Toledo LL, Ferreira MCM, Rodrigues LOC, Rezende NA. Neurofibromatose tipo 1: mais comum e grave do que se imagina. Rev Assoc Med Bras. 2009;55(4):394-9.

34. Marques AC, Dinis F. Neurofibromatose tipo 1: relato de um caso clínico. Rev Port Med Geral Fam. 2013;29(5):322-6. Disponível em: http://www.scielo.mec.pt/pdf/rpmgf/v29n5/ v29n5a07.pdf.

35 Avila DS, Pando CS, Meneghello LP, Ramos TN. Neurofibromatose tipo 1. Rev AMRIGS. 2010;54(3):317-20.

36 Espig AF, Slomp AA, Campagnolo AQ, Rockenbach DM, Silva BD, Pomblum VJ. Neurofibromatose tipo 1: atualização. Rev Bras Clin Med. 2008;6:243-9.

37 Moraes FS, Santos WEM, Salomão GH. Neurofibromatose tipo I. Rev Bras Oftalmol. 2013;72(2):128-31. Disponível em: http://www.scielo.br/pdf/rbof/v72n2/13.pdf.

Submetido em: 15.11 .17

Aceito em: 05.04.18 